\title{
Desarrollo histórico de los indicadores de Ciencia y Tecnología, avances en América Latina y México
}

\author{
Edna Alcázar Farías*, Alejandro Lozano Guzmán**
}

Resumen: Los indicadores de Ciencia y Tecnología son de gran utilidad en el desarrollo de políticas adecuadas de investigación. La información que éstos proporcionan permite realizar una asignación correcta de los recursos, otorgar financiación, tomar decisiones en la gestión de grupos y de proyectos, y, mediante la determinación de capacidades y la identificación de debilidades, orientar mejor a la investigación.

La evaluación con indicadores de ciencia y tecnología es ampliamente utilizada, de acuerdo con métodos empleados y aceptados internacionalmente, los cuales se han desarrollado desde los años treinta y se han adaptado a las necesidades de cada época. En el presente resumen se realiza una revisión de los principales avances que sobre indicadores de ciencia y tecnología se han tenido hasta la fecha. Así mismo se hace énfasis en la necesidad de desarrollar nuevas metodologías de evaluación del impacto socioeconómico de la ciencia y la tecnología.

Palabras clave: evaluación, impacto económico, impacto social, indicador, innovación.

\section{Historical development of Science and Technology Indicators advances in Latin America and Mexico}

Abstract: Science and technology (SET) indicators are very useful in the development of research policies. They assist decision-makers in distributing resources appropriately, in the awarding of funding, in the management of research groups and projects, and, via the identification of capacities and weaknesses, better orientate research. These indicators are widely used, following internationally accepted methodologies that have been developed since the 1930s and adapted to the needs of different times. However, the relationship between engineering, technology and economic activity has insufficient indicators and there is a lack of methodologies for investigating it; the accurate assessment of the social, educational, cultural, environmental and commercial impact(s) of engineering projects is very difficult. With the aim of being able to determine the impact of research and technological development, this work discusses the main advances made in the use of SET indicators. Emphasis is placed on the need to develop new methodologies for assessing the socioeconomic impact of science and technology, particularly from the perspective of Latin American countries.

Keywords: assessment, economic impact, indicator, innovation, social impact.

* División de Investigación y Posgrado, Facultad de Ingeniería, Universidad Autónoma de Querétaro, México. Correo-e: ednaalcazar@uaq.mx.

** Consejo de Ciencia y Tecnología del Estado de Querétaro, México. Correo-e: alcctq@concyteq. org. $m x$.

Recibido: 5-8-08; 2. ${ }^{a}$ versión: 24-12-08; 3. ${ }^{a}$ versión: 15-4-09. 


\section{Introducción}

La ciencia, la tecnología, y la innovación, tienen un papel protagonista en el crecimiento económico, en la productividad, en la competitividad, en el desarrollo sostenible y en el mejoramiento de la calidad de vida de una sociedad. Desde esta perspectiva, es fundamental la elaboración de políticas, por parte de los Gobiernos de cada país, que orienten la dirección que ha de tener la investigación, de acuerdo con las necesidades y capacidades propias de cada región, de tal manera que se desarrollen, fortalezcan y mantengan las capacidades científicas adquiridas.

En la construcción de estas políticas debe contarse con datos precisos que reflejen el impacto y los alcances de las actividades científicas y tecnológicas en una sociedad, en lo económico, en lo académico, así como en el medio ambiente. Con esta información, los países, especialmente los que están en vías de desarrollo, tendrían las bases necesarias para justificar el incremento de su inversión en ciencia y tecnología, a un nivel que eleve el desarrollo socioeconómico y reduzca la cada vez mayor brecha científica y tecnológica con los países desarrollados.

Sin una correcta medición, no se puede planificar, prever e innovar. Por ello, el presente documento tiene como una de sus finalidades describir, con base en los antecedentes históricos, la evolución de los métodos evaluativos de la ciencia y la tecnología. Asimismo, se enfatiza sobre la importancia y necesidad de medir el impacto económico y social generado por la investigación, y sobre los problemas y limitaciones que presentan las metodologías utilizadas.

\section{Evolución del desarrollo de indicadores de ciencia y tecnología}

\subsection{0 a 1960}

En 1930 la Unión Soviética fue el primer país que utilizó información estadística sobre ciencia y tecnología, con la finalidad de impulsarlas como un recurso al servicio de la nación. Posteriormente, en 1940, los Estados Unidos comenzaron a recopilar los primeros datos estadísticos sobre esta actividad.

Después de la II Guerra Mundial se reconoce el impacto que la ciencia tiene en el desarrollo económico de las naciones, y a iniciativa de los Estados dominantes y de algunas instituciones internacionales — como la Organización de las Naciones Unidas para la Educación, la Ciencia y la Cultura (UNESCO), la National Science Foundation (NSF) y la Organización para la Cooperación y el Desarrollo Económico (OCDE) - el tema de los indicadores de evaluación toma gran fuerza y, así, varios países llevan a cabo sus propias estadísticas. Pero, dadas las distintas metodologías de obtención de datos, resultaban incomparables debido a la diferencia de conceptos y criterios utilizados. 


\subsection{Década de los años sesenta}

Destaca el trabajo de instituciones norteamericanas privadas como el Institute for Scientific Information (ISI), que fue creado en 1961 por Eugene Garfield ${ }^{1}$, y el Computer Horizon Incorporation (creado a finales de la década de 1960), entre otros, que han desarrollado técnicas y métodos de análisis de la ciencia.

En 1963 la OCDE edita el primer manual de lo que después se conocería como la Familia Frascati, destinado a describir el método a seguir para realizar encuestas que permitieran obtener datos sobre el personal dedicado a la investigación y al desarrollo experimental (OCDE, 1963).

\subsection{Década de los años setenta}

En 1972 el National Science Board (NSB) de la National Science Foundation (NSF) escribió la primera edición de Science and Engineering Indicators, publicación que se ha convertido en una de las referencias más comunes del análisis cuantitativo de la ciencia (NSB, 1989).

\subsection{Década de los años ochenta}

En la mayoría de los países europeos se generaliza la tendencia de evaluar el impacto de la ciencia y la tecnología con el inicio de las operaciones de las agencias dedicadas a la evaluación de tipo variado. En Reino Unido, Holanda y Francia, por ejemplo, se desarrollan técnicas de medición cuantitativa, destacando autores como Martin e Irvine (1984), Callon (1987), Rip (1987), Leydesdorff (1990a, 1990b) y Van Raan (1993), quienes se han centrado en el análisis de indicadores de salida. En Australia se impulsa la producción de indicadores específicos para cada institución de investigación, apoyada por el Australian Research Council (Velho, 1994). Asimismo, la OCDE publica un Suplemento del Manual de Frascati, destinado a medir los resultados en la enseñanza superior y a la preparación de estadísticas de investigación y desarrollo.

\subsection{Década de los años noventa}

En 1990 la OCDE publica el Manual de la Balanza de Pagos Tecnológicos $(B P T)$, (proporciona pautas para analizar las transacciones comerciales relacionadas con el conocimiento científico y tecnológico de un país con el resto del mundo) y en 1992 la presenta el Manual de Oslo (sistematiza las mediciones so-

\footnotetext{
${ }^{1}$ Posteriormente en 1992, fue adquirida por Thomson Business Information, y actualmente es conocido como Thomson ISI.
} 
bre innovación tecnológica utilizando un modelo interactivo de relación en cadena del proceso de innovación, el cual destaca el papel que los gobiernos pueden representar para promover la innovación a través del tejido económico). En 1994 el Manual de Patentes (mide las transferencias de tecnología a los sectores productivos mediante el registro de patentes), se incorpora a esta línea de trabajo, completando así a la Familia Frascati.

El Manual de Canberra, el cual define un marco teórico que sirve de guía para recopilar datos estadísticos comparables internacionalmente en relación con la existencia y demanda de personal dedicado a ciencia y tecnología, se publica en 1995 como un trabajo conjunto entre la OCDE y Eurotat.

América Latina se incorpora en esta década al proceso de desarrollo de indicadores de evaluación. A partir del trabajo previo de numerosos investigadores de diversos países, en 1995 se sientan las bases para la creación de la Red Iberoamericana e Interamericana de Ciencia y Tecnología (RICYT), organismo que ha trabajado para el logro de una Red de Indicadores de Ciencia y Tecnología que considere la perspectiva de Latinoamérica.

En México, toma importancia la construcción de indicadores de Ciencia y Tecnología. Desde 1991, el CONACYT viene presentando su Informe General del Estado de la Ciencia y la Tecnología en México, y desde 1996 ha dado a conocer la información referente a indicadores y estadísticas del país, así como su ubicación con respecto a otras naciones, en una publicación anual titulada Indicadores de Actividades Científicas y Tecnológicas, que presenta tres tipos principales de indicadores: el gasto en ciencia y tecnología; el acervo de recursos humanos en ciencia y tecnología; y la producción científica y tecnológica, y su impacto económico.

En esta misma década vuelve a tomar importancia el tema de la evaluación del impacto de la ciencia y la tecnología, tal y como lo señalan autores como Kostoff (1995), Oszlak y O'Donnell (1995) quienes apoyan la importancia de contar con indicadores de impacto con el objeto de obtener mayor conocimiento acerca de los distintos impactos de la ciencia y tecnología y, por otra parte, para apoyar la toma de decisión en política científica y tecnológica, especialmente en la evaluación y asignación de recursos a proyectos o unidades de investigación.

\subsection{Primera década del nuevo milenio}

De acuerdo con el Banco Interamericano de Desarrollo (BID, 2006), el impacto de la ciencia y la tecnología es observable y puede ser medido en tres ámbitos principales: en el conocimiento, en lo económico y en lo social. El primero es medido mediante indicadores bibliométricos, las citas recibidas, las publicaciones y las patentes. Para el segundo impacto es posible utilizar la balanza de pagos de tecnología, el comercio de bienes de alta tecnología, y la innovación tecnológica. En el caso de la medición de los impactos sociales, hasta el momento no existen indicadores completamente normalizados. 
Algunas de las principales metodologías reconocidas de evaluación de impacto socioeconómico de las actividades de ciencia y tecnología, son las empleadas por la OCDE, por Invertec, por Melkes de la Universidad de Chicago, la evaluación por pares, la evaluación costo-beneficio, los indicadores sectoriales, y el método de producción e impacto. Además hay otros métodos, que varían desde una simple lista o modelos de ponderación, hasta los modelos complejos de programación matemática y de simulación.

Godin (2005a), acepta que los indicadores de ciencia y tecnología se han vuelto una medida de la modernidad y del progreso de las sociedades y los países. En su investigación admite que la ciencia se ha convertido en una "empresa" que cuenta con importantes recursos financieros e intelectuales, que influye directamente sobre las acciones de la sociedad. Lo cual da importancia a la medida de la ciencia, y justifica que desde hace décadas expertos universitarios y organismos estadísticos nacionales midan a los científicos y sus actividades, con la esperanza de optimizar y orientar a las políticas y a las actividades científicas. En Godin (2005b), se destaca la importancia que está adquiriendo la demanda de estadísticas del impacto social y económico de la ciencia, la tecnología y la innovación. Este autor sugiere que para construir estos nuevos indicadores se requiere de nuevas cuestiones conceptuales, nuevas metodologías y nuevas personas o investigadores que estén más inmersos en los nuevos marcos históricos de la sociedad moderna.

Ya en esta década se reconoce el trabajo realizado por la RICYT en el desarrollo de nuevos indicadores, ejemplos del trabajo de este organismo son la publicación, en 2001, del Manual de Normalización de Indicadores de Innovación Tecnológica en América Latina y el Caribe (Manual de Bogotá) y en 2006 del Manual de Lisboa. En el primero se realiza una conceptualización de la situación de la región, y en el segundo se proponen las pautas para la interpretación y el análisis de los datos estadísticos disponibles, así como la construcción de indicadores referidos a las tecnologías de la información y las comunicaciones.

En México la evaluación del impacto de la ciencia y la tecnología adquiere mayor importancia, en este sentido la Ley de Ciencia y Tecnología, subraya la necesidad de realizar evaluaciones periódicas sobre las actividades de investigación y desarrollo tecnológico que hayan recibido fondos públicos. Lo anterior resulta especialmente importante dentro las instituciones que aplican este tipo de recursos en actividades científicas, con la finalidad de impulsar una asignación de recursos más eficiente, y a la vez justificar la inversión en ciencia y tecnología ante la sociedad.

A pesar de los esfuerzos realizados, el BID señala que los sistemas de información en América Latina son débiles y dispersos, debido a la limitada disponibilidad de datos confiables relacionados con tendencias en ciencia, tecnología e innovación, habiendo huecos en los informes y dudas en cuanto a la confiabilidad y definiciones, lo que dificulta un análisis profundo de las necesidades y del progreso, y limita, además, el desarrollo de estrategias certeras de investigación. 


\subsection{Retos futuros}

A nivel mundial, entre los retos de la OCDE en esta nueva etapa (Gault, 2007) se encuentran el desarrollo de un sistema de Indicadores de Ciencia y Tecnología comparable al sistema de indicadores financieros existentes. Asimismo, este organismo tiene como retos presentar un indicador compuesto de ciencia, tecnología e innovación, y además fortalecer las políticas científicas. En este sentido, se destaca la importancia de los indicadores en el monitoreo, mercadeo, previsión y evaluación del gasto de los recursos públicos en programas y proyectos.

De acuerdo con OCDE, los indicadores que probablemente tomarán mayor importancia serán: indicadores de actividades (investigación y desarrollo, invención, innovación, difusión del conocimiento y la tecnología, desarrollo de recursos humanos); indicadores de enlace (contratos, colaboración, comercialización, redes, fuentes de conocimiento, tecnologías y prácticas); indicadores de resultados (mercado, empleos especializados, ganancias), e indicadores de impacto (forma en que la ciencia y la tecnología han cambiado las condiciones de la sociedad).

\section{Conclusiones}

La evaluación del impacto real de la ciencia y la tecnología debe ser considerada como un asunto central, con la finalidad de construir políticas científicas y tecnológicas acertadas, que produzcan innovación y que den solución a los principales problemas que caracterizan a los países en desarrollo. Los criterios hasta ahora utilizados para evaluar el mérito científico en los países en desarrollo, no son completamente adecuados para la toma de decisiones de políticas de investigación, al igual que no son adecuadas para otorgar financiación correctamente dirigida. En este sentido, es necesario elaborar nuevas metodologías que consideren la relación entre los recursos invertidos en ciencia y tecnología y sus productos, en términos de los efectos reales registrados sobre la competitividad industrial, el crecimiento de la economía, la mejor calidad de vida de la sociedad, el empleo, la educación, la cultura y el medio ambiente, por mencionar algunas.

En América Latina y en México, una metodología de evaluación sobre el impacto de las actividades científicas y tecnológicas, más acorde con las condiciones internas prevalecientes, aportaría una base más sólida sobre la que sería posible definir propósitos generales y particulares, objetivos y metas, y evaluar el comportamiento y las repercusiones de la ciencia y la tecnología. Aún más, esta nueva metodología sería de utilidad para evaluar los efectos, en sus diferentes dimensiones, de la investigación desarrollada en el pasado, y por supuesto para determinar los alcances de la ciencia y la tecnología que se desarrolla en el presente. El resultado de este análisis sería de utilidad a la hora de definir el tipo de actividades científicas y tecnológicas más apropiadas para el futuro. 
Considerando los antecedentes históricos presentados en este trabajo, la metodología de evaluación sobre el impacto de las actividades de investigación científica y tecnológica que es deseable aplicar en los países en desarrollo, es imprescindible que considere las problemáticas locales para que, si menoscabo de la excelencia académica, sirva para resolver problemas locales. De esta manera se evitará perpetuar el sistema de dependencia "país desarrollado-en vías de desarrollo" en el que la investigación de "estado del conocimiento" llevada a cabo por estos últimos países, sirve en muchas ocasiones, para que desde los países desarrollados se envíen productos y servicios de alto contenido científico y tecnológico a los países en vías de desarrollo, con un alto costo económico y social para estos últimos.

En la nueva metodología, también deberá hacerse énfasis en dar a conocer a la sociedad los productos de la investigación, ya que esto ayudaría a legitimar, sostener y fortalecer el sistema de ciencia, tecnología e innovación de los países en vías de desarrollo.

\section{Bibliografía}

Bid (2006): Educación, ciencia y tecnología en América Latina y el Caribe: Un compendio estadístico de indicadores. Departamento de Desarrollo Sostenible Interamericano del Desarrollo. Washington D.C.

Callon, M. (1987): Society in the making: The study of technology as a tool for sociological analysis. En W. Bijker, T. Hughes and T. Pinch (eds.), The Social Construction of Technological Systems, p 83-103. London: MIT Press.

Gault, F. (2007): Science, Technology and Innovation Indicators in the OECD: Next Steps. VII Congreso Iberoamericano de Indicadores de Ciencia y Tecnología. Red de Indicadores de Ciencia y Tecnología (RICYT). Iberoamericana e Interamericana. Sao Paulo, Brasil, 23 al 25 de mayo. http://www.ricyt.org/interior/interior.asp?Nivel1=6\& Nivel2=2\&Id [7 de junio del 2008.]

Godin, B. (2005a): La science sous observation: cent ans de mesures sur les scientifiques 1906-2006. Presses de l'Université Laval. ISBN 2-7637-8297-3. 96 pp. Québec, Canada.

Godin, B. (2005b): Measurement of Science and Technology: 1920 to the Present. Routledge. ISBN 0-4153-4104-3. 360 pp. London and New York.

Kostoff, R. (1995): The handbook of research impact assessment. Office of Naval Research. Arlington VA.

Ley de Ciencia y Tecnología, artículo 12, fracciones I y VIII. http://www.diputados.gob. $\mathrm{mx} /$ LeyesBiblio/242.pdf [16/01/2008].

Leydesdorff, L. (1990a): Relation among science indicators or more generally predictions on the basis of anything one might know about texts. The dynamics of science. Scientometrics, 19, 271-296.

Leydesdorff, L. (1990b): The prediction of science indicators using information theory. Scientometrics, 19, 297-324. 
Martin, B., e Irvine, J. (1984): CERN: past performance and future prospects I. CERN's position in world high-energy physics. Research Policy, 13, 183-210.

NSB (1989): Science \& engineering indicators. Washington, D.C.

OCDE (1963): The measurement of Science and Technological Activities. Proposed standard practice for surveys of Research and Experimental Development. "Frascati Manual Frascati. OCDE. 261 pp. París.

Oszlak, O., y O'Donnell, G. (1995): Estado y políticas estatales en América Latina. En la sección Dossier de REDES: Revista de Estudios Sociales de la Ciencia, 2 (4), septiembre. Buenos Aires.

Rip, A. (1987): The challenges to science policy studies. Metascience, 5, 75-92.

Van Raan, A. (1993): Advanced bibliometric methods to assess research performance and scientific development: basic principles and recent practical applications. Research Evaluation. 3, 151-166.

Velho, L. (1994): Indicadores científicos: aspectos teóricos y metodológicos. En Martínez, E. (ed.), Ciencia, tecnología y desarrollo: interrelaciones teóricas y metodológicas. Nueva Sociedad. Caracas. UNESCO. 